\title{
How to Suppress Virus from Propagating Efficiently
}

\author{
Wang Siwei \\ School of Information Science and Technology, Fudan University, Shanghai 200433, China \\ email: 12307130057@fudan.edu.cn
}

Keywords: Scale Free Network, SIS Model, Immunization

\begin{abstract}
The SI, SIR and SIS model were introduced at first. And then do the simulation based on various immunizations. The model of network will choose the scale free network and the use SIS model to simulate the propagation of the virus, some guidance in maintaining are obtained. More than $27.5 \%$ of the total nodes are protected under improved acquaintance immunizations, the virus can be suppressed perfectly but need a bit longer time. Double acquaintance immunization could protect both the selected nodes and neighbors but shorten running time. In order to verify the conclusion more visually, computer simulation will be used instead of mathematical calculation.
\end{abstract}

\section{Introduction}

A complex network is a graph which consists of a gigantic number of nodes and the way the nodes are connected with each other is completely unpredictable, thus creating a network with an extremely complicated topological structure. The complex network can reflect the real world and the relationships around us in everyday life better than those regular networks. The small world network model [1] and the scale free network model [2] are researched most among those complex networks. Researching the propagation principle of the complex network is of great importance, for the propagation is fairly usual in natural world. If we can understand the principle abstractly, it will be much easier for us to suppress the propagation of virus in computer network or the rumor among ordinary people in order to make the least effort to prevent the virus from spreading rapidly.

\section{The virus propagation model}

According to the latest research, the most accepted and popular models of virus propagation are the SI model, SIR model and SIS model, each of which has its own feature and calculating methods [3].

In SI model, once an individual is infected, it is infected permanently. In SIR model, after an individual is infected, it will be removed from the network with the velocity of $\gamma$ which means the individual is either cured and has obtained the immunity to certain disease or maybe the worst situation, death. In SIS model, the individual will come back to susceptible mode gradually after being infected, which is the primary difference to the SIR model. The SIS model is close to most daily situation. A computer may be infected by an attack of a virus in certain period of time and then successfully remove the virus thanks to antivirus software or other methods but it can still be infected by another mutated virus. The SIS model is more suitable for long-term virus propagation. SIS model was chosen to research in the scale free network.

\section{The methods to suppress the virus}

In order to suppress the propagation of virus, countless methods had been used. The random immunization is fast and easy to realize indeed, but its effectiveness is not so good. The targeted immunization is one of the most efficient strategies proved. However, it needs all round topological structure of the graph which is not feasible in this growing network. The acquaintance immunization avoids the difficulty in acquiring the topology of the whole network and has a relatively good effect [4]. 


\section{The description of the spread of virus in the light of SIS model and the immunization}

During every period of time in history when a certain virus burst out, there is always a tiny individual or a small community which was infected initially. The virus would then infect its susceptible neighbors gradually. The basic idea of either an individual or a small neighbor is infected at first is identical.

Some prerequisite is needed to assume to build the SIS model. Suppose that only an individual is infected at the very beginning of the infection, the scale of the network is finite [5]. Create an array called infected $[\mathrm{N}]$ to record those infected nodes, and another one called immune [N] to record the nodes which are protected. Select certain number of the nodes randomly by randomizing a group of different numbers ranging from zero to $\mathrm{N}-1$ ( $\mathrm{N}$ means the amount of nodes in the network). Carry out the various immune strategies. If the node $\mathrm{i}$ is protected, assign 1 to immune [i].

In the SIS model, there are two parameters called $\beta$ and $\gamma . \beta$ means the probability that one node infects it virulence to its neighbor. $\gamma$ means the probability that an infected node becomes susceptible one. We introduce $\lambda=\beta / \gamma$ [6] called effective propagation rate to indicate the spreading velocity of a virus.

In order to control the least variables as well as drawing the correct answer, it is logical to fix the value of $\gamma$ to 0.8 and change to value of $\beta$ solely which will only affect the time scale of the spreading of virus. The total number of nodes is 4000 for every new nodes, it connects out two edges with those previous nodes which already exist.

\section{The propagation of virus without immunization}

Simulate propagation of the virus in scale free network. In the first experiment, infect the node that has an average degree. Place $\beta$ as $0.0375,0.1,0.125$ and 0.25 , according to the features of the scale free network, a correct tendency should be that the larger the value of $\beta$ is, the faster the velocity of the infection of the network will be. The simulation result is shown in figure 1.

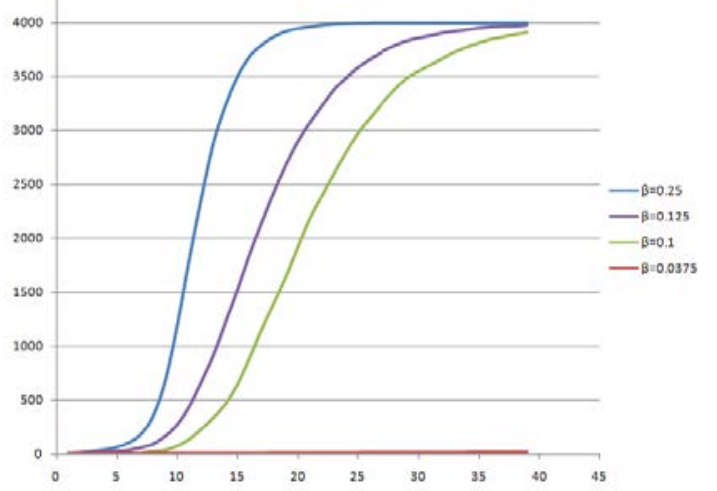

Fig.1 relationship between steps and infected nodes

As is shown the small the $\beta$, the slow the velocity. It means the lower infect probability of one node, the lower the virus to infect other nodes.

Fix the value $\beta$ as 0.125 and vary initial node which is infected at the very beginning, if those nodes are attacked, the speed of the propagation of the virus will be faster at the beginning shown in figure 2 . 


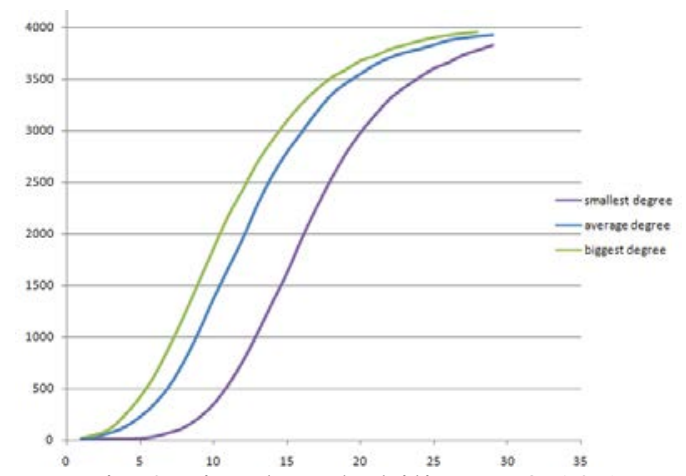

Fig.2 Fixed probability as 0.125

\section{The propagation of virus with different immunization strategy}

Acquaintance immunization. Acquaintance immunization has a big advantage. The immunization strategy does not need to know the topological structure of the whole network which is much more practical than the targeted immunization [7]. The total number of nodes is still 4000 at $\beta$ as 0.4 and protect 1200 nodes, under the same condition, the bigger node infected, the bigger velocity of virus infection or the slope of the curve shown in figure 3 .

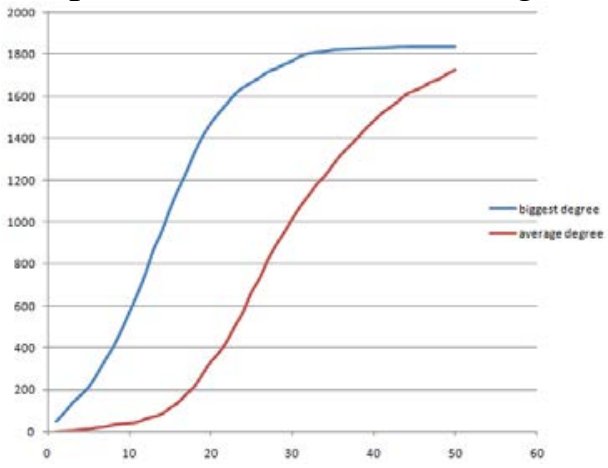

Fig.3 Acquaintance Immunization

Improved Acquaintance Immunization. Acquaintance immunization does not make sure it could always choose the neighbor with the biggest degree. If the node with the biggest degree is chosen to protect, the virus can be suppressed more effectively, it is named improved acquaintance immunization, sign as IAI. The IAI makes sure chosen node has the biggest degree among all the neighbors of one certain randomly selected. If node collective has two different biggest degree nodes, continuing searching the node with the second biggest degree until find the node which differs from the previous nodes.

Shown in figure 4, the immunization is more effective than acquaintance immunization, the protect efficiency grows rapidly with the increase of the number of immune nodes, protected number increased to some near 1100, the immune efficiency would be extraordinarily perfect, in other words, more than $27.5 \%$ of the total nodes are protected, the virus can be suppressed perfectly. It provides a feasible method to antivirus strategy.

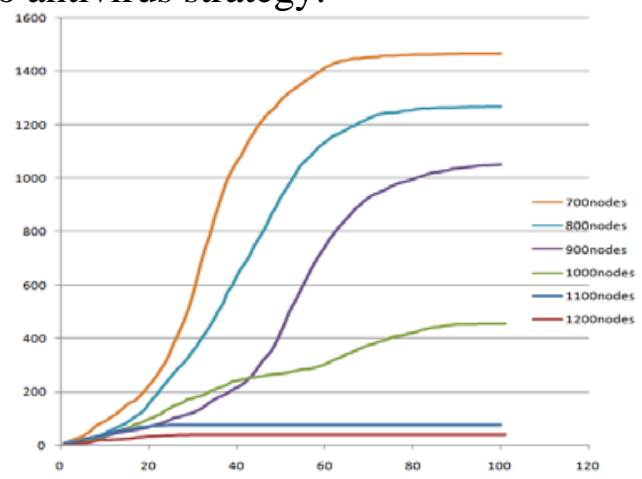

Fig.4 Improved Acquaintance Immunization 
Double Acquaintance Immunization. The improved acquaintance immunization is more effective but average running time longer. Double acquaintance immunization (DAI) strategy also selects several nodes randomly, protects both the selected ones and the neighbors with the biggest degree of them and not the network, it shorten the running time. It is showed in figure 5.

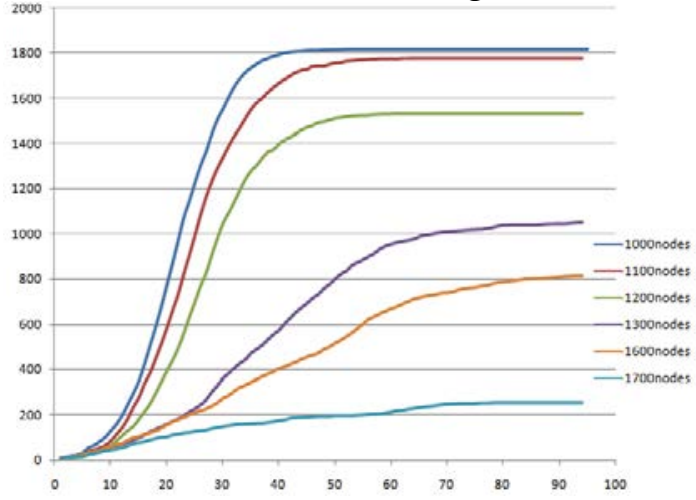

Fig.5 Double Acquaintance Immunization

The DAI is a feasible method to suppress the virus from propagation. It is shown in figure 6 for 1300 nodes and figure 7 for 1700 nodes. The result means that if a virus erupts, it could take less time to protect the appropriate nodes than the IAI does. That means antivirus program would take less time to spread into the network.

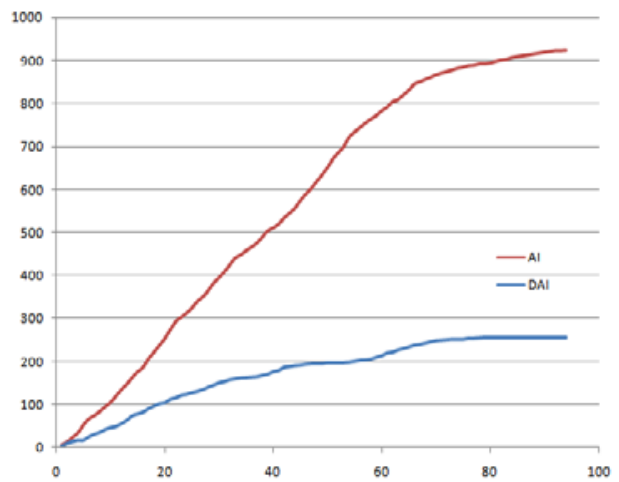

Fig.6 Compare between DAI and IAI in 1300 nodes

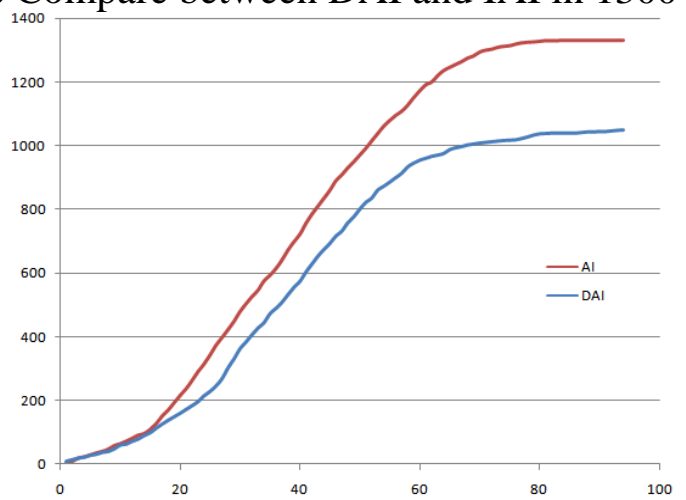

Fig.7 Compare between DAI and IAI in 1700 nodes

\section{Conclusions and prospect}

The paper has chosen SIS model as numerical simulation virus propagation model after comparing three models of SI, SIR and SIS, proposed acquaintance immunization, improved acquaintance immunization and double acquaintance immunization using in networks for antivirus under the assumption of different nodes, and the virus propagation performance was predicted. It provides a feasible method to antivirus strategy.

(1) For propagation of virus without immunization, the lower infect probability of one node, the lower the virus to infect other nodes.

(2) Under the same condition, the bigger node infected, the bigger velocity of virus infection 
depend on the acquaintance.

(3) More than $27.5 \%$ of the total nodes are protected under improved acquaintance immunizations, the virus can be suppressed perfectly but need a bit longer time.

(4) Double acquaintance immunization could protect both the selected nodes and neighbors but shorten running time.

This is only the result on simulation. In real network the topological structure is much more complicated. It is not absolute but still feasible and efficiency, it can still give us some guidance in maintaining the network and eliminate the computer virus. In the field of complex network, more research needs to be taken in revealing the essence of it.

\section{Acknowledgements}

This work was financially supported by Special Fund for Science and Technology Commission of Shanghai Municipality innovation action plan (13dz1203701).

\section{Reference}

[1] D. J. Watts and S. H. Strogatz. Collective dynamics of 'small worlds' networks. Nature, 393(6684),1998:440-442

[2] Barabasi A L, Albert R. Emergence of scaling in random networks [J]. Science, 1999, 286:509-512

[3] T. Zhou, Z. Q. Fu, and B. H. Wang. Epidemic dynamics on complex networks. Arxiv preprint physics/0508096,2005.

[4] Pastor-Satorras and Vespignani. Epidemics and immunization in scale free networks, page 113-132. Wiley-VCH, 2002.

[5] Romualdo Pastor-Satorras. Epidemic dynamics in finite size scale free networks. Physical Review E, Volume 65, 035108 (R). 2002

[6] Wang Xiaofan, Li Xiang, Chen Guanrong. The theory of complex network and its application [M]. Beijing: Tsinghua University press, 2011,3

[7] N. Madar, T. Kalisky, R. Cohen, D. ben Avraham, and S. Havlin. Immunization and epidemic dynamics in complex networks. The European Physical Journal B-Condensed Matter, 38(2):169-276, 2004 